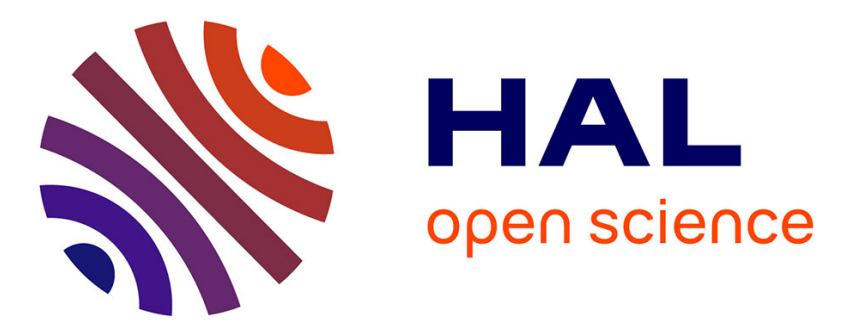

\title{
Ribose and related sugars from ultraviolet irradiation of interstellar ice analogues
}

\author{
Cornelia Meinert, Iuliia Myrgorodska, Pierre de Marcellus, Thomas Buhse, \\ Laurent Nahon, Søren V. Hoffmann, Louis Le Sergeant D’hendecourt, Uwe J.
} Meierhenrich

\section{To cite this version:}

Cornelia Meinert, Iuliia Myrgorodska, Pierre de Marcellus, Thomas Buhse, Laurent Nahon, et al.. Ribose and related sugars from ultraviolet irradiation of interstellar ice analogues. Science, 2016, 10.1126/science.aad8137 . hal-01278288v2

\section{HAL Id: hal-01278288 \\ https://hal.science/hal-01278288v2}

Submitted on 18 Apr 2016

HAL is a multi-disciplinary open access archive for the deposit and dissemination of scientific research documents, whether they are published or not. The documents may come from teaching and research institutions in France or abroad, or from public or private research centers.
L'archive ouverte pluridisciplinaire HAL, est destinée au dépôt et à la diffusion de documents scientifiques de niveau recherche, publiés ou non, émanant des établissements d'enseignement et de recherche français ou étrangers, des laboratoires publics ou privés.

\section{(1) (1) $\$$}

Distributed under a Creative Commons Attribution - NonCommercial - NoDerivatives| 4.0 


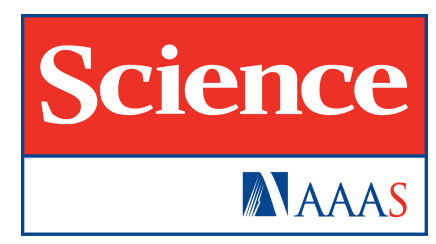

\section{Supplementary Materials for}

Ribose and related sugars from ultraviolet irradiation of interstellar ice analogues

Cornelia Meinert, ${ }^{1}$ luliia Myrgorodska, ${ }^{1,2}$ Pierre de Marcellus, ${ }^{3}$ Thomas Buhse, ${ }^{4}$ Laurent Nahon, ${ }^{2}$ Soeren V. Hoffmann, ${ }^{5}$ Louis Le Sergeant d'Hendecourt, ${ }^{3}$ Uwe J. Meierhenrich ${ }^{1}$

correspondence to: Cornelia.Meinert@unice.fr and Uwe.Meierhenrich@unice.fr

\section{This PDF file includes:}

Materials and Methods

Figs. S1 to S6

Table S1

Caption for Movie S1

Other Supplementary Materials for this manuscript includes the following:

Movie S1 


\section{Materials and Methods}

Preparation of the interstellar pre-cometary ice analogue

The experimental setup has already been described in detail elsewhere (30). It consists of a high vacuum chamber $\left(10^{-7} \mathrm{mbar}\right)$ in which an infrared (IR) transparent $\mathrm{MgF}_{2}$ window is cooled down to $78 \mathrm{~K}$. A gas mixture, previously prepared in an independent stainless steel gas line pumped down to about $5.10^{-6} \mathrm{mbar}$, is then injected into the chamber where it condenses on the cold $\mathrm{MgF}_{2}$ substrate to form a thin film of "ices". These ices were, simultaneously to their deposition, irradiated by UV photons for $142 \mathrm{~h}$, using an $\mathrm{H}_{2}$ discharge lamp providing essentially Lyman- $\alpha$ photons at $122 \mathrm{~nm}$ with a tail including an $\mathrm{H}_{2}$ recombination line at approximately $160 \mathrm{~nm}$ and a continuum down to the visible range. In our experiments the ratio of ultraviolet photons to deposited molecules is around 1 , meaning approximately $10 \mathrm{eV}$ per deposited molecule to obtain the soluble organic matter sample. The sample was further irradiated, at room temperature, with right-hand circularly polarized synchrotron radiation (CPSR) at $10.2 \mathrm{eV}$ for $2 \mathrm{~h}$ at the beamline DESIRS (31) of the SOLEIL synchrotron because we initially intended to induce asymmetric photochemical reactions. At this level of interpretation, no stereochemical effects induced by CPSR could be definitely identified yet in our samples. The entire experiment in terms of deposition rate, ice mixture, and the effect of UV photolysis is constantly monitored by IR spectroscopy which reveals the presence of a few photochemical produced molecules and radicals such as $\mathrm{CO}, \mathrm{CO}_{2}$, $\mathrm{HCO}$, and $\mathrm{H}_{2} \mathrm{CO}$, trapped in the ice phase. Photon-molecule interactions are assumed to occur predominantly in the condensed phase but in rare events UV photons may have initiated gas-phase reactions, with the photo-products being subsequently trapped on the ices.

For this study, we prepared one mixture composed of $\mathrm{H}_{2} \mathrm{O},{ }^{13} \mathrm{CH}_{3} \mathrm{OH}$, and $\mathrm{NH}_{3}$ in relative proportions 10:3.5:1, qualitatively representative of interstellar/pre-cometary ices. Methanol, our only source of carbon, was labelled with ${ }^{13} \mathrm{C}$ in order to avoid any confusion with potential biological contamination in the handling and analysis processes of the samples. $\mathrm{H}_{2} \mathrm{O}$ (water, liquid) was purified by using a Millipore Direct Q5 system, ${ }^{13} \mathrm{CH}_{3} \mathrm{OH}$ (methanol, liquid) was purchased from Aldrich (99.9\% purity), and $\mathrm{NH}_{3}$ (ammonia, gas) from Messer (99.98\% purity). The ratios between the components were determined by their partial pressures in the gas line, measured by an absolute pressure gauge (Baratron). The physico-chemical state of the initial sample (temperature and ice composition) is used as a template of pre-accretionary ices and is not fully representative of interstellar conditions. At $78 \mathrm{~K}$, enhanced diffusion of reactants allows for a faster chemical evolution than at $10 \mathrm{~K}$ without significantly affecting the nature of the organic residue (32). The organic residue is always extracted after warm-up to room temperature as when considering the chemical composition of meteorites. For the initial molecular ice composition, we observed that $\mathrm{CO}$ and $\mathrm{CO}_{2}{ }^{13} \mathrm{C}$ isotopologues appear during the photochemical process, formed and trapped in the ice and are thus part of the icy molecular chemical reservoir. During the preparation of the samples infrared monitoring is used to control deposition rate and UV efficiency by using the apparition of a weak $\mathrm{HCO}$ band and a reasonably strong $\mathrm{H}_{2} \mathrm{CO}$ band, which falls at $1720 \mathrm{~cm}^{-1}$. The $3.4-\mu \mathrm{m}$ feature, which consists of two subfeatures at $2925 \mathrm{~cm}^{-1}(3.42 \mu \mathrm{m})$ and $2875 \mathrm{~cm}^{-1}$ $(3.48 \mu \mathrm{m})$ indicative to the presence of $\mathrm{CH}_{2} \mathrm{OH}$ groups (33), was recorded in our ice 
during temperature increase to $230 \mathrm{~K}$ when the sublimation of water, methanol, and ammonia is almost finished. Below $230 \mathrm{~K}$ the sample is optically too thick for the detection of the $3.4-\mu \mathrm{m}$ feature.

Analysis of the interstellar pre-cometary ice analogue

Sample-handling glassware was wrapped in aluminum foil, and heated at $500{ }^{\circ} \mathrm{C}$ for $3 \mathrm{~h}$ prior to usage. Eppendorf tips ${ }^{\circledR}$ were sterile and the water used for extraction, standard solutions, reagent solutions, and blanks had been prepared by a Milipore Super$\mathrm{Q}$ water filter system (4 ppb total organic carbon). The organic residues were extracted with $150 \mu \mathrm{L}$ high-performance liquid chromatography grade water from their $\mathrm{MgF}_{2}$ windows and transferred into conical reaction vials. The samples were silylated with

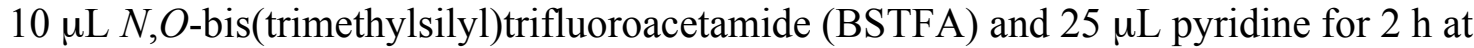
$80^{\circ} \mathrm{C}$. The samples were evaporated to dryness using a gentle stream of nitrogen and $30 \mu \mathrm{L}$ of the internal standard methyl laurate in $n$-hexane $\left(5 \cdot 10^{-5} \mathrm{M}\right)$ were added. The mixtures were transferred into $\mathrm{GC}$ vials prior their analyses by two-dimensional gas chromatography-time-of-flight mass spectrometry ( $\mathrm{GC} \times \mathrm{GC}-\mathrm{TOFMS})$ using a LECO Pegasus IV D system. Procedural blanks were run in sequence to each sample in order to monitor significant background interferences.

The $\mathrm{GC} \times \mathrm{GC}$ column set consisted of a Chirasil-Dex chemically bonded primary column $(24.5 \mathrm{~m} \times 0.25 \mathrm{~mm}$ inner diameter (ID), $0.25 \mu \mathrm{m}$ film thickness) modulatorcoupled to an Agilent Wax secondary column $(1.40 \mathrm{~m} \times 0.1 \mathrm{~mm}$ ID, $0.1 \mu \mathrm{m}$ film thickness). Helium was used as carrier gas at a constant flow rate $\bar{u}=1 \mathrm{~mL} \mathrm{m^{-1 }}$. Sample volumes of $1 \mu \mathrm{L}$ were injected in the splitless mode at an injector temperature of $230^{\circ} \mathrm{C}$. The primary oven was operated as follows: $40^{\circ} \mathrm{C}$ for $1 \mathrm{~min}$, temperature increase of $10{ }^{\circ} \mathrm{C} \mathrm{min}{ }^{-1}$ to $80{ }^{\circ} \mathrm{C}$ and held for $15 \mathrm{~min}$, followed by $2{ }^{\circ} \mathrm{C} \mathrm{min}^{-1}$ to $180{ }^{\circ} \mathrm{C}$, and an isothermal hold at $180^{\circ} \mathrm{C}$ for $5 \mathrm{~min}$. The secondary oven used the same temperature program with a constant temperature offset of $15^{\circ} \mathrm{C}$. A modulation period of 3 seconds was applied. The TOF-MS was operated at a storage rate of $150 \mathrm{~Hz}$, with a 25-500 amu mass range. Data were acquired and processed with LECO Corp ChromaTOF ${ }^{\mathrm{TM}}$ software. Compound identification was performed by comparison with the chromatographic retention in both dimensions and mass spectra of authentic standards. All reference compounds were purchased from Sigma Aldrich including the monosaccharides: glycolaldehyde dimer, D,L-, D-, and L-glyceraldehyde, dihydroxyacetone, erythrose, erythrulose, ribose, arabinose, lyxose, xylose, and ribulose; the saccarinic acids: glyceric and threonic acid; the polyols: ethylene glycol, 1,3-propanediol, glycerol, threitol, erythritol, xylitol, arabitol, mannitol, sorbitol, and dulcitol; the hydroxycarboxylic acids: glycolic, D,L-; D-; and L-lactic, 3-hydroxypropanoic, 2-hydroxyisobutyric, S-2-hydroxybutyric, S-3-hydroxybutyric, 4-hydroxybutyric, malic, dicarboxylic acid: succinic, and fumaric acid as well as the dialdehyde: glyoxal. The mass spectra of unknown analytes were interpreted based on their fragmentation patterns. Compounds were quantified using external standards.

Arabitol can be formed by the reduction of either arabinose or lyxose. Therefore, arabitol is sometimes but less often called lyxitol. In Figure 1 we used the name arabitol for both, the reduced arabinose and for the reduced lyxose. 
Analyte quantification

The simulated interstellar pre-cometary ice samples were deposited onto magnesium fluoride $\left(\mathrm{MgF}_{2}\right)$ windows. The condensation was performed on two rectangles of $4 \mathrm{~mm}$ to $9 \mathrm{~mm}$ providing a sample surface $A=72 \mathrm{~mm}^{2}$ and a sample mass $m=100 \pm 50 \mu \mathrm{g}$. To avoid sample loss while weighting, the mass and its estimated error were deduced from IR spectroscopy by comparison with a similar sample previously measured as a standard at the Institute d'Astrophysique Spatiale (IAS) (34). The quantification of each analyte indicated in ppm by mass (Figure 1) corresponds to the mass of the analyte in a $100-\mu \mathrm{g}$ ice sample. Relative quantities from one molecular species to another are reliable to within the indicated error bars. Absolute quantities vary within $\pm 50 \%$. Note however that the absolute errors are less important if one considers the final conclusion that the detected sugars are abundant within this sample.

The quantities of the analytes were calculated based on the relative response factors $(R R F)$ using standard solutions with known concentration of the internal standard and the compound of interest. The $R R F$ was calculated as follows: $R R F=A_{\mathrm{X}}{ }^{\cdot} C_{\mathrm{IS}} / A_{\mathrm{I}}{ }^{\circ} c_{\mathrm{X}}$, where $A_{\mathrm{X}}$ is the peak area of the analyte, $A_{\mathrm{IS}}$ the peak area of the internal standard, $c_{\mathrm{X}}$ and $c_{\mathrm{IS}}$ are the concentrations of the analyte and internal standard, respectively. The approximations for the compounds without corresponding standard were based on the $R R F$ of structurally related compounds, i.e. $R R F($ threonic acid $)=R R F($ erythronic acid $)=R R F($ ribonic acid $)=$ $R R F($ lyxonic acid $)=R R F($ yylonic acid $) ; R R F($ ribulose $)=R R F($ xylulose $)$, and $R R F($ xylitol $)=R R F($ ribitol $)$.

The $R R F \mathrm{~s}$ of ribose, arabinose, lyxose and xylose were calculated for mass/charge $(\mathrm{m} / \mathrm{z}) 204\left({ }^{12} \mathrm{C}\right)$ and $206\left({ }^{13} \mathrm{C}\right)$, respectively, to avoid any contribution from co-eluting compounds. For xylulose and ribulose, $\mathrm{m} / \mathrm{z} 335$ and 339, respectively, were used for the same reason. As for the other analytes, the peak area and $R R F \mathrm{~s}$ were calculated for the most abundant mass fragment $m / z$ 73. For arabinose, lyxose, and xylose the sum of both anomeric forms ( $\alpha$ - and $\beta$-pyranose) was used. The calculated concentrations of the identified sugar and sugar-related compounds (Fig. 2) accompanied by their experimental $3 \sigma$ errors $(n=6)$ are given in Table S1.

Figure S1 provides a supplementary view on the multidimensional gas chromatographic separation of ribose and other monosaccharides and sugar-related molecules. Potential ${ }^{13} \mathrm{C}$-contamination from biological and/or laboratory sources was determined by analyzing a blank sample. Therefore, $150 \mu \mathrm{L}$ HPLC grade water was dried in a conical reaction vial, derivatized with BSTFA and pyridine, and analyzed under identical analytical conditions as the pre-cometary ice sample. Figure S2 shows the $\mathrm{GC} \times \mathrm{GC}-\mathrm{TOFMS}$ chromatogram of the blank sample, in which monosaccharides are clearly absent.

Identification of organic molecules in the interstellar pre-cometary ice analogue

Compound identification was performed by comparison with the chromatographic retention in both dimensions of authentic standards and mass spectra. For those compounds that were not available as standard solution, e.g. 3-hydroxyisobutyric acid, we utilized the mass selectivity of the fragments of silylated derivatives, provided by the characteristic electron impact (EI) mass spectra, present in the NIST library (35).

Comparison with previously reported data on the photoproducts formed in the organic residues of interstellar ice mixtures (10) let us identify oxamic acid (36). 
2-Hydroxymethylglycerol - one of the major products in the photochemically induced formose reaction - was identified from its reported spectral and analytical data (19) as well as 2-hydroxymethyltetritol (20).

Identification of monosacharides in the interstellar pre-cometary ice analogue

Monosaccharides with an anomeric center can yield mixtures of $\alpha$ - and $\beta$-anomeric derivatives. The possibility of both pyranose (6-membered ring) and furanose (5-membered ring) forms of sugar molecules increases the number of potential derivatives. Mass spectra, however, allow pyranose derivatives to be unambiguously distinguished from furanose derivatives $(37,38)$. Pyranose trimethylsilyl ethers produce characteristic ions by electron impact sugar degradation at: $\mathrm{m} / \mathrm{z} 217\left({ }^{13} \mathrm{C}=220\right)$ $\left[\left(\mathrm{CH}_{3}\right)_{3} \mathrm{SiOCH}=\mathrm{CH}-\mathrm{CH}=\mathrm{OSi}\left(\mathrm{CH}_{3}\right)_{3}\right]^{+}, \mathrm{m} / \mathrm{z} 204\left({ }^{13} \mathrm{C}=206\right)$ $\left[\left(\mathrm{CH}_{3}\right)_{3} \mathrm{SiOCH}=\mathrm{CHOSi}\left(\mathrm{CH}_{3}\right)_{3}\right]^{+*}$, and $\mathrm{m} / \mathrm{z} 191\left({ }^{13} \mathrm{C}=192\right)\left[\left(\mathrm{CH}_{3}\right)_{3} \mathrm{SiOCH}=\mathrm{OSi}\left(\mathrm{CH}_{3}\right)_{3}\right]^{+}$. The ion at $m / \mathrm{z} 217\left({ }^{13} \mathrm{C}=220\right)$ is generally very intense for the furanose form whereas the relative intensity of the ion at $m / z 204\left({ }^{13} \mathrm{C}=206\right)$ is always less than $5 \%$. Based on our recorded mass spectra we thus conclude that the majority of the extracted and derivatized C5-sugar molecules from the interstellar ice sample is present in the pyranose ring form. Since the known fragmentation pattern for trimethylsilyl derivatives is almost identical for all of the C5-sugar pyranosides, the assignment was confirmed by the retention time and comparison with authentic standards. We used the following observations to distinguish between the $\alpha$ - and $\beta$-anomeric derivatives. Figure S3 shows the mass spectra and the characteristic mass fragmentation pathway of ribose, representative for all other $\mathrm{C}-5$ monosaccharides.

10 Rules for monosaccharides identification

1. Anomerization occurring in pyridine during preparation of TMS derivatives is minimal (39).

2. Each anomer yields one peak. All free $\mathrm{OH}$ groups are thus converted to TMS derivatives (39).

3. $\alpha$ - and $\beta$-composition of equilibrium mixtures of sugars in a) aqueous solution: arabinose: $\alpha=51 \%, \beta=44 \%$; lyxose: $\alpha=74 \%, \beta=26 \%$; ribose: $\alpha=89 \%$ \& xylose: $\alpha=41 \%, \beta=55 \%$ and in b) pyridine: arabinose: $\alpha=32 \%, \beta=53 \%$; lyxose: $\alpha=72 \%$, $\beta=28 \%$; ribose: $\alpha=19 \%, \beta=63 \%$ \& xylose: $\alpha=46 \%, \beta=43 \%(39,40)$.

4. Excellent resolution of anomeric forms. Structural properties imparted by pyranose and furanose ring account for their excellent separation of various stereoisomers.

5. $\alpha$ - and $\beta$-pyranose separation factor: ribose $(1.08)<$ arabinose $(1.2)<$ lyxose $(1.4)<$ xylose $(1.4)(39,40)$.

6. $\alpha$-Anomer elutes before $\beta$-anomer in case of glucose, galactose, mannose, talose, xylose, lyxose, and arabinose $(39,41)$.

7. Pyranosides having planar structures are more strongly retained (39). The first peaks eluted are always $\alpha$-lyxo- and $\alpha$-arabinopyranoses; they are also the less planar pyranose forms. Maximum retention is always attained for $\beta$-xylose, whose TMS groups are all equatorial.

8. Elution order of pyranoses: $\alpha$-lyxose $\approx \alpha$-arabinose $<\beta$-arabinose $=\beta$-ribose $<$ $\beta$-lyxose $\approx \alpha$-ribose $<\alpha$-xylose $<\beta$-xylose (41). 
9. Retention times of ketoses in ring form are often lower than those of the analogues aldoses whereas the open form (ketol) is more strongly retained (42).

10. Replacement of $\mathrm{OH}$ by $\mathrm{H}$ in deoxysugars leads to decreased relative retention times (39).

Identification of sugar alcohols in the interstellar pre-cometary ice analogue

The trimethylsilyl derivatives of alditols with three to six carbon atoms including glycerol, erythritol, threitol, arabinitol, glucitol, xylitol, ribitol, galactitol, and mannitol that were all detected in the organic residue of the simulated interstellar ices - were studied in detail (43). The mass spectra and corresponding fragmentation pattern of arabitol (arabinitol) are illustrated in Figure S4. Favoured $\alpha$-cleavage with charge retention followed by rearrangement loss of trimethylsilanol $\left[\left(\left(\mathrm{CH}_{3}\right)_{3}\right) \mathrm{Si}-\mathrm{OH}\right]^{+}$has been shown as the predominant fragmentation pathway. For the alditols this type of resonance stabilization favors cleavage of all other $\mathrm{C}$ - $\mathrm{C}$ bonds. The abundance of these $\alpha$-cleavage ions is explained by the stabilization of the positive charge by the unshared electron pairs on the oxygen atoms. The differentiation of diastereomeric derivatives, however, is nearly impossible due to the small differences between these spectra.

For sugar alcohols that were not commercially available, we compared their reported retention indices with the proposed structures detected in our study with $\mathrm{GC} \times \mathrm{GC}$ TOFMS. The elution order and final peak assignment was thus possible for the $\mathrm{C}-5$ alditols: ribitol and alditol (42) as well as for the C-6 sugar alcohols: allitol and talitol (altritol) (39).

Identification of saccharinic, hydroxycarboxylic, and dicarboxylic acids in the interstellar pre-cometary ice analogue

Fragmentation studies on the aliphatic hydroxycarboxylic acids $(44,45)$ glycolic acid, lactic acid, and 2-hydroxybutyric acid:

- $m / z=147$ : through rearrangement decomposition of the $[\mathrm{M}-15]^{+}$ions, which can be described as cyclic oxonim ions and subsequent loss of $\mathrm{CO}$ and $\mathrm{CH}_{2} \mathrm{O}$ for glycolic acid and $\mathrm{CO}$ and $\mathrm{CH}_{3} \mathrm{CHO}$ for lactic acid

- $m / z=147$ : for 2-hydroxybutyric acids direct migration of the ester trimethylsiloxyl group to a positive charge site at the other silicon atom

Fragmentation studies on the aliphatic dicarboxylic \& hydroxy dicarboxylic acids (45) succininc acid and malic acid:

- the molecular ion $[\mathrm{M}]^{\bullet+}=262\left(266\right.$ for $\left.{ }^{13} \mathrm{C}\right)$ and $[\mathrm{M}]^{\bullet+}=350(354)$ - often absent in trimethylsilyl derivatives - is observed for succinic and malic acid, respectively

Fragmentation studies on the saccharinic acids (44) glyceric acid, erythronic acid, threonic, lyxonic, ribonic (Figure S5), xylonic and arabinoic acid:

- $m / z=292$ (294): all acids with $\mathrm{OH}$ in C2 and C3 position; McLafferty-type rearrangement (ester migration) of a trimethylsilyl group with aldehyde loss (2-deoxyaldonic acid disclose that formation)

- $m / z=205$ (207): indicative of a vicinal diol group

- [M-15-90]: glyceric acid 217 (220) and erythronic acid 319 (323) 
- $m / z=189$ (191): characteristic for glyceric acid by stepwise loss from [M$15]^{+}$of both trimethylsilanol and carbon monoxide [M-15-118]

Fragmentation studies on C5 sugar acids:

- $m / z=307$ (310): decomposes by loss of formaldehyde to $m / z=277$ (279)

- $m / z=307$ (310): decomposes by rearrangement loss of trimethylsilanol to $m / z=217(220)$

- $m / z=423$ (427): metastable peak formed from loss of the end group with electron withdrawing effect of the ester group: $[\mathrm{M}]^{\bullet+}=527(532)-[103 / 104-$ $\mathrm{H}]$, further decomposes to $\mathrm{m} / \mathrm{z}=333$ (337) by loss of trimethylsilanol

Fragmentation studies on the branched-chain saccharinic acid (44) 2-methylglyceric acid:

- $\quad[\mathrm{M}-15]: 321(325)$

- $m / z=306$ (308): McLafferty-type rearrangement

- $m / z=233$ (236) \& $m / z=219$ (222): chain cleavage ions with charge retention at $\mathrm{C} 2$ 
Fig. S1

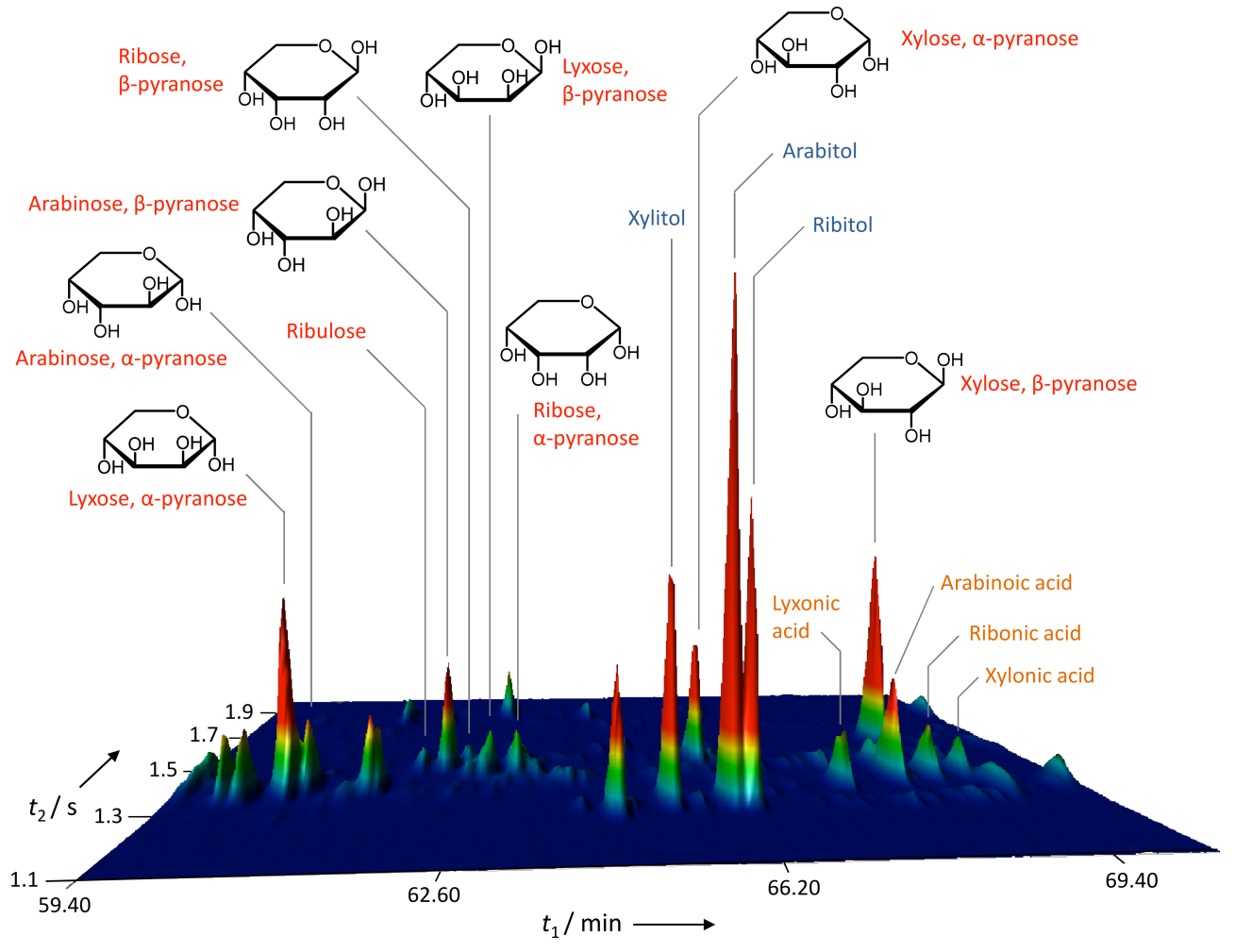

Fig. S1. Supplementary view on the multidimensional gas chromatographic separation of ribose and other monosaccharides and sugar-related molecules. Experimental conditions are identical as compared to Figure 2. This view better allows for the visual separation of the $\alpha$ - and $\beta$-pyranose of xylose and arabinoic acid. Atomic mass units 206 and 294 were selected for the above representation with $z$-scaling $=10^{5}$. 
Fig. $\mathbf{S 2}$

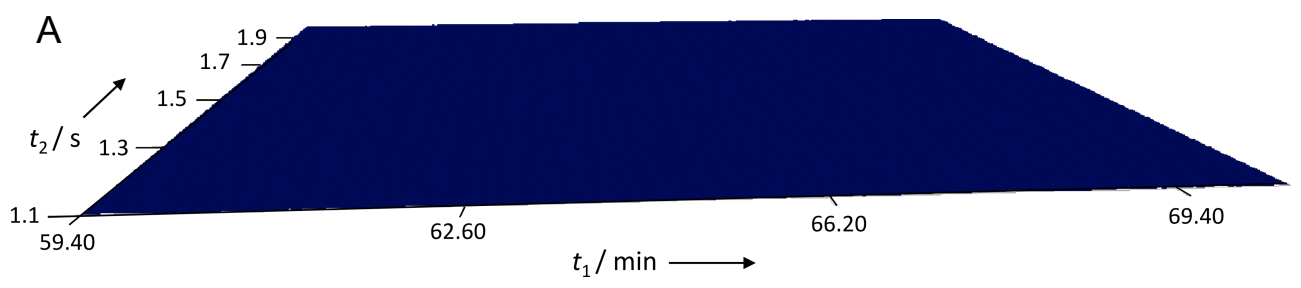

B

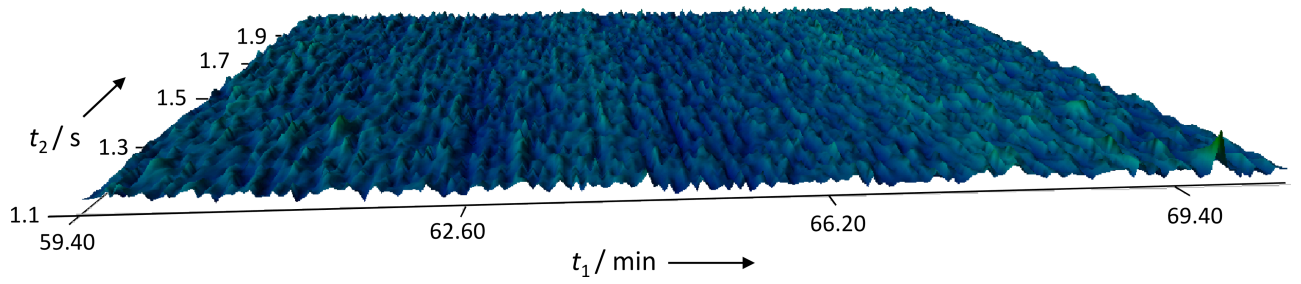

Fig. S2. Analytical blank. A: Representative enantioselective $\mathrm{GC} \times \mathrm{GC}-\mathrm{TOFMS}$ chromatogram of the blank sample submitted to the entire analytical protocol. Atomic mass units 206 and $294(\times 5)$ were selected for the above representation and the primary and secondary temperature program was identical to Figure S1. B: Reduced $z$-scaling $(z$-axis $=5,000)$ illustrates the absence of monosaccharides and other related compounds in the blank sample. 
Fig. S3

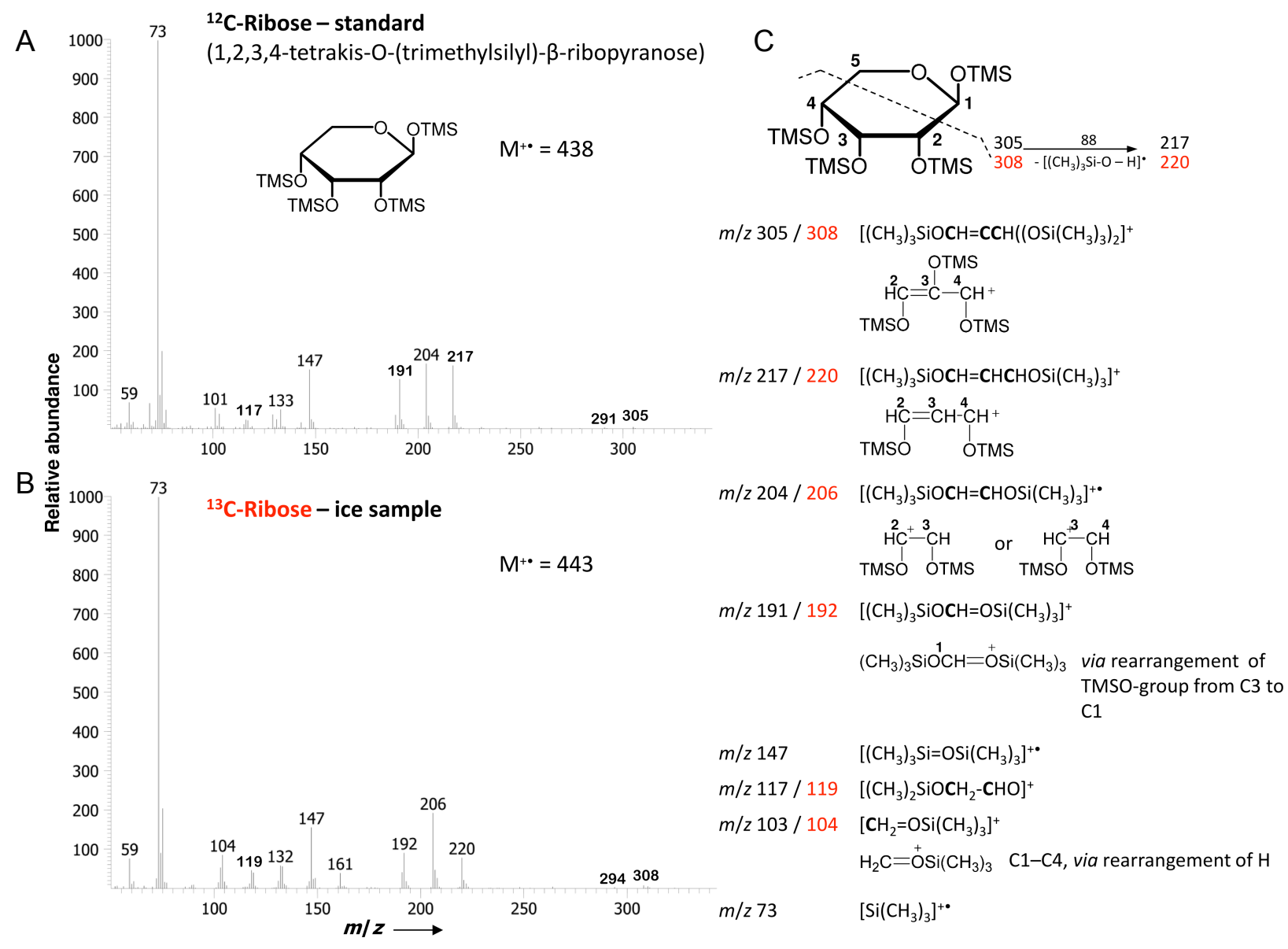

Fig. S3. Mass spectra of the ${ }^{12} \mathrm{C}$-ribose standard (A) and the ${ }^{13} \mathrm{C}$-ribose (B) identified in the pre-cometary ice sample. The ribose fragmentation pattern is indicated (C) by representing the individual mass shifts for each fragment of the ${ }^{12} \mathrm{C}$ standard (black) and the ${ }^{13} \mathrm{C}$ sample (red). 
Fig. S4

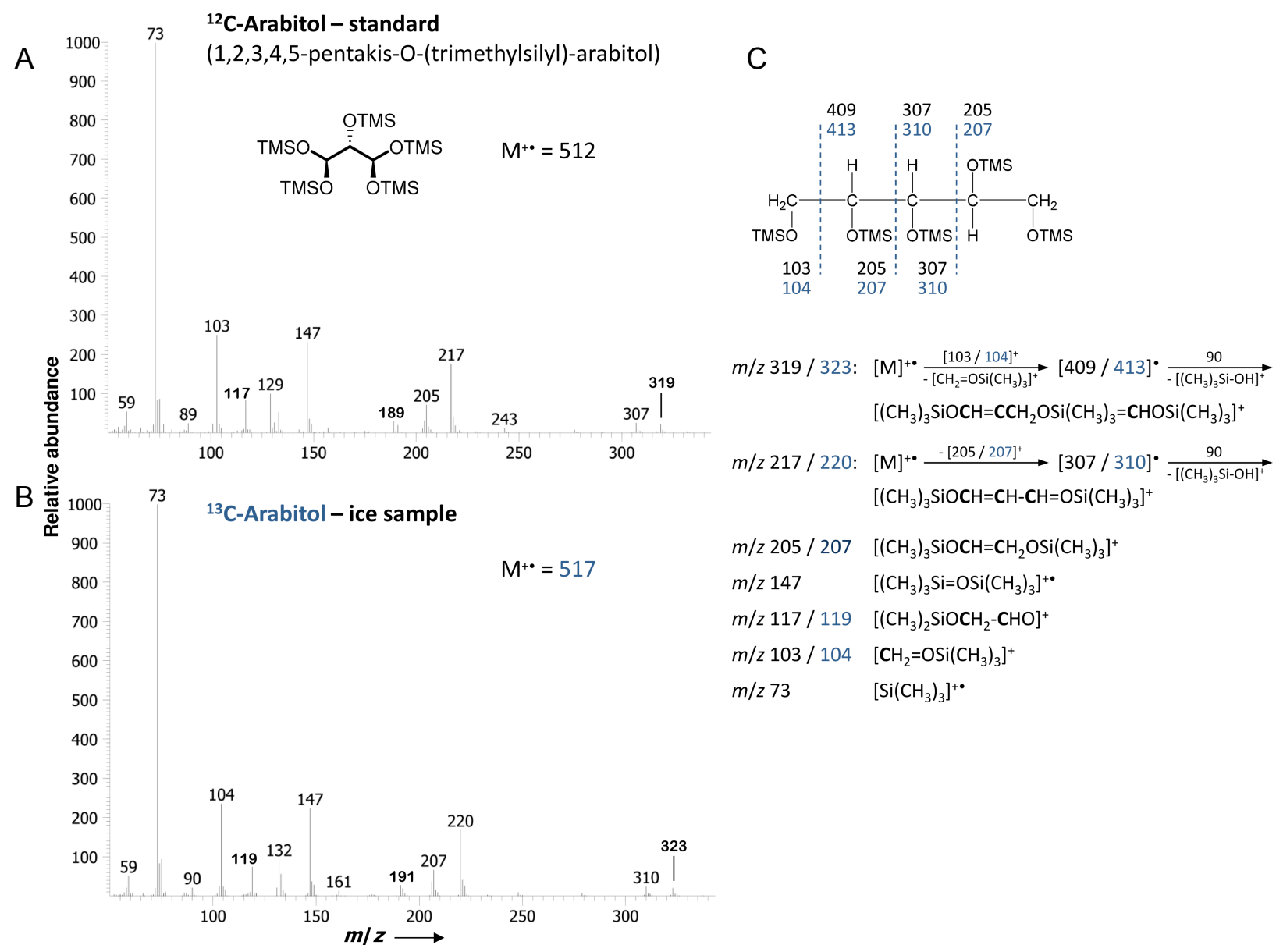

Fig. S4. Mass spectra of the ${ }^{12} \mathrm{C}$-arabitol standard $(\mathrm{A})$ and the ${ }^{13} \mathrm{C}$-arabitol ice sample (B). The arabitol fragmentation pattern is indicated (C) by representing the individual mass shifts for each fragment of the ${ }^{12} \mathrm{C}$ standard (black) and the ${ }^{13} \mathrm{C}$ sample (blue). 
Fig. 55

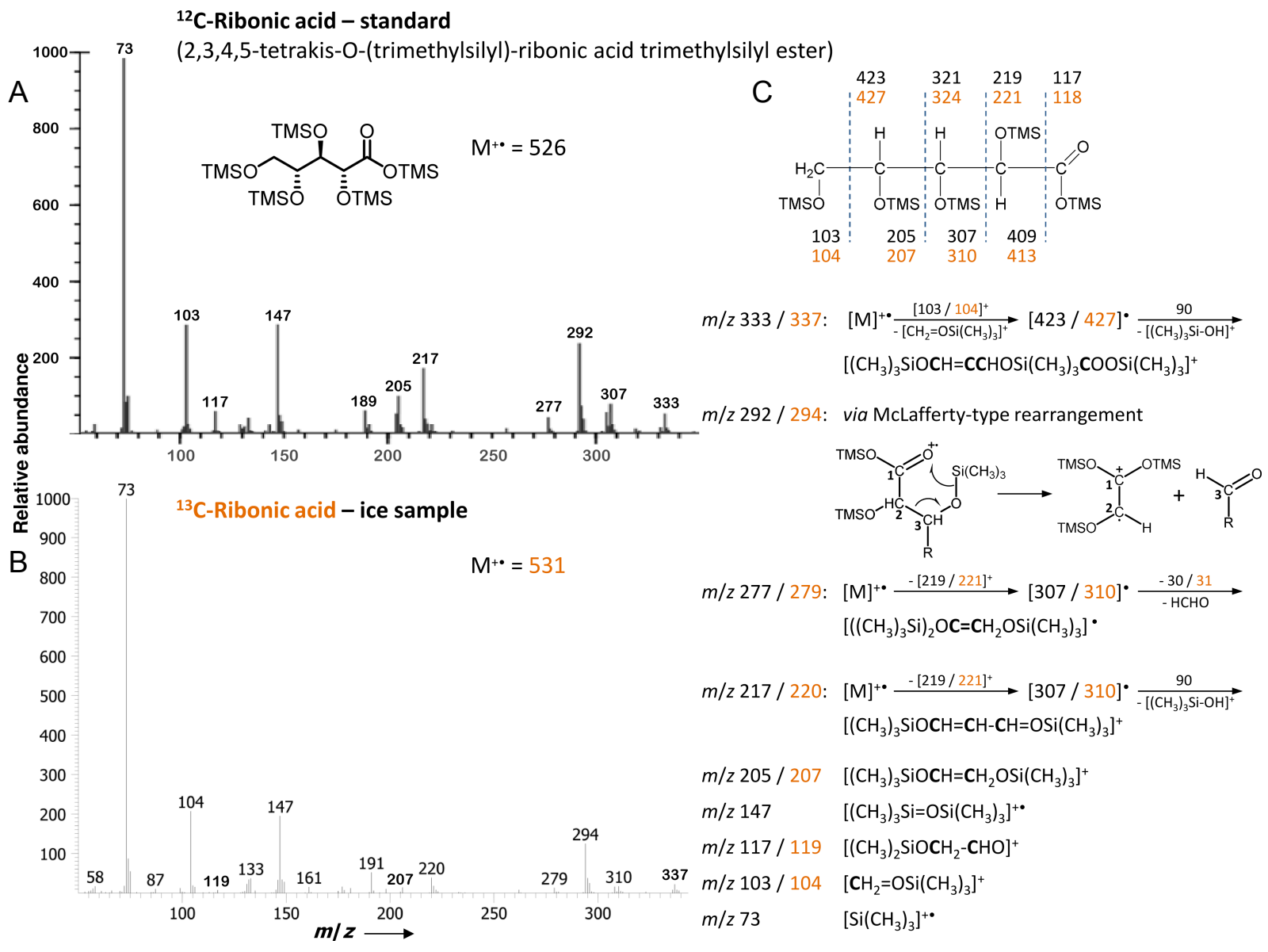

Fig. S5. Mass spectra of the ${ }^{12} \mathrm{C}$-ribonic acid standard (A) and the ${ }^{13} \mathrm{C}$-ribonic acid in the pre-cometary ice sample (B). The ribonic acid fragmentation pattern is indicated (C) by representing the individual mass shifts for each fragment of the ${ }^{12} \mathrm{C}$ standard (black) and the ${ }^{13} \mathrm{C}$ sample (orange). The mass spectrum of ribonic acid was taken from the NIST database (35). 
Fig. S6

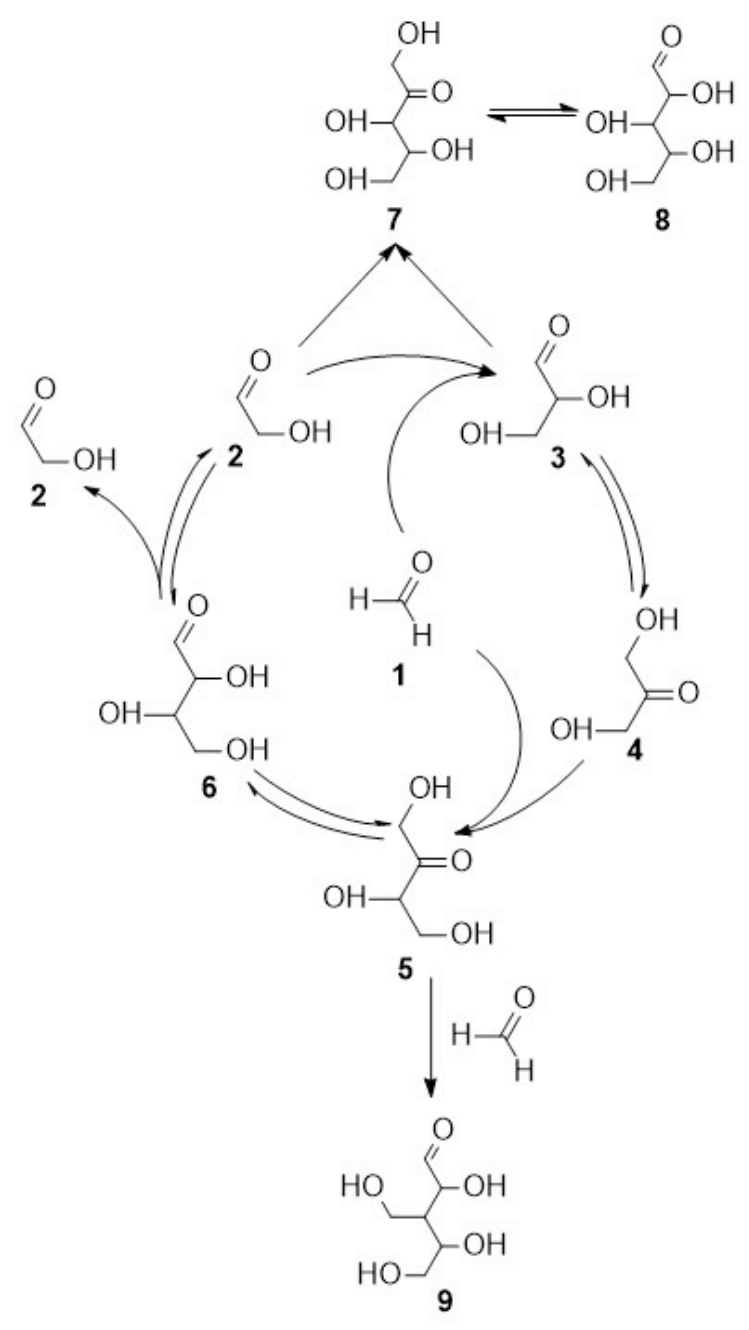

Fig. S6. The formose reaction. Formaldehyde 1 undergoes self-condensation under autocatalytic reaction kinetics to form glycolaldehyde $\mathbf{2}$, which undergoes an aldol reaction with an equivalent of $\mathbf{1}$ by forming glyceraldehyde 3 . Dihydroxyacetone 4 is formed by aldose-ketose isomerization, a tautomerization, of 3 . Dihydroxyacetone 4 reacts with 1 producing ketotetrose (erythrulose) 5 and aldotetrose $\mathbf{6}$ (18). The aldol condensation of $\mathbf{2}$ and $\mathbf{4}$ forms the 2-ketopentoses $\mathbf{7}$ that isomerize to an aldopentose $\mathbf{8}$ such as ribose, arabinose, xylose, and lyxose. Branched aldotetroses 9 are formed by aldol condensation of $\mathbf{5}$ and $\mathbf{1}$. 


\section{Table S1.}

Table S1 Summary of the total abundances of sugar and sugar-related compounds in the water extract of a pre-cometary interstellar ice analogue. [a] Quantity of carbon atoms. [b] The data are mean values given with their standard deviation of $3 \sigma$.

\begin{tabular}{|c|c|c|c|c|c|}
\hline$\# C^{[a]}$ & Compound & $\begin{array}{l}\text { Quantity } \\
\text { [ppm] }\end{array}$ & $\# C^{[a]}$ & Compound & $\begin{array}{l}\text { Quantity } \\
\text { [ppm] }\end{array}$ \\
\hline \multicolumn{3}{|c|}{ Aldoses / aldehydes } & \multicolumn{3}{|c|}{ Ketoses } \\
\hline 2 & Glycolaldehyde & $2390 \pm 100$ & 2 & - & \\
\hline 3 & D,L-Glyceraldehyde & $302 \pm 30$ & 3 & Dihydroxyacetone & $540 \pm 40$ \\
\hline 4 & D,L-Erythrose & LOQ* & 4 & Erythrulose & $37 \pm 4$ \\
\hline \multirow[t]{4}{*}{5} & D,L-Ribose & $260 \pm 20$ & 5 & Ribulose & $2010 \pm 110$ \\
\hline & D,L-Arabinose & $200 \pm 20$ & & Xylulose & $470 \pm 50$ \\
\hline & D,L-Xylose & $240 \pm 15$ & & & \\
\hline & D,L-Lyxose & $145 \pm 11$ & & & \\
\hline \multicolumn{3}{|c|}{ Sugar acids (saccharinic acids) } & \multicolumn{3}{|c|}{ Sugar alcohols / polyols } \\
\hline 2 & Glycolic acid & $6330 \pm 750$ & 2 & Ethylene glycol & $550 \pm 50$ \\
\hline 3 & D,L-Glyceric acid & $2440 \pm 30$ & 3 & Glycerol & $2860 \pm 90$ \\
\hline \multirow[t]{2}{*}{4} & D,L-Erythronic acid & $960 \pm 40$ & 4 & Erythritol & $5070 \pm 500$ \\
\hline & D,L-Threonic acid & $840 \pm 50$ & & D,L-Threitol & $7200 \pm 680$ \\
\hline \multirow[t]{4}{*}{5} & D,L-Ribonic acid & $82 \pm 7$ & 5 & D,L-Ribitol & $560 \pm 50$ \\
\hline & D,L-Arabinoic acid & $165 \pm 9$ & & D,L-Xylitol & $630 \pm 40$ \\
\hline & D,L-Xylonic acid & $67 \pm 5$ & & D,L-Arabitol & $1150 \pm 90$ \\
\hline & D,L-Lyxonic acid & $140 \pm 15$ & & & \\
\hline
\end{tabular}

[*] Limit of quantification. ['] The low relative abundance of ethylene glycol as compared to the corresponding C2 aldehyde and $\mathrm{C} 2$ acid is due to its high volatility resulting in its loss during sample preparation and analysis. 


\section{Movie S1}

Movie S1 provides a supplementary view on the multidimensional gas chromatographic separation of ribose and other monosaccharides and sugar-related molecules.

\section{References of the Supplementary Materials:}

30. M. Nuevo et al., Enantiomeric separation of complex organic molecules from irradiation of interstellar/circumstellar ice analogs. Adv. Space Res. 39, 400-404 (2007).

31. L. Nahon et al., DESIRS: a state-of-the-art VUV beamline featuring high resolution and variable polarization for spectroscopy and dichroism at SOLEIL. J. Synchtrotron R. 19, 508520 (2012).

32. G. M. Muñoz Caro, W. A. Schutte, UV-photoprocessing of interstellar ice analogs: New infrared spectroscopic results. Astron. Astrophys. 412, 121-132 (2003).

33. G. M. Muñoz Caro, E. Dartois, A tracer of organic matter of prebiotic interest in space, made from UV and thermal processing of ice mantles. Astron. Astrophys. 494, 109-115 (2009).

34. P. Modica et al., Enantiomeric excesses induced in amino acids by ultraviolet circularly polarized light irradiation of extraterrestrial ice analogs: a possible source of asymmetry for prebiotic chemistry. Astrophys. J. 788, 79 (2014).

35. S. E. Stein, in NIST Chemistry WebBook, NIST Standard Reference Database Number 69, P. J. Linstrom, W. G. Mallard, Eds. (National Institute of Standards and Technology, Gaithersburg, MD).

36. H. Horai et al., MassBank: a public repository for sharing mass spectral data for life sciences. J. Mass Spectrom. 45, 703-714 (2010).

37. D. C. DeJongh et al., Analysis of trimethylsilyl derivatives of carbohydrates by gas chromatography and mass spectrometry. J. Am. Chem. Soc. 91, 1728-1740 (1969).

38. J. Lönngren, S. Svensson, Mass spectrometry in structural analysis of natural carbohydrates. Adv. Carbohydr. Chem. Biochem. 29, 41-106 (1974).

39. C. C. Sweeley, R. Bentley, M. Makita, W. W. Wells, Gas-liquid chromatography of trimethylsilyl derivatives of sugars and related substances. J. Am. Chem. Soc. 85, 2497-2507 (1963).

40. M. Paez et al., Identification of the components of aldoses in a tautomeric equilibrium mixture as their trimethylsilyl ethers by capillary gas chromatography. Chromatographia 23, 43-46 (1987).

41. A. García-Raso et al., Gas chromatographic behavior of carbohydrate trimethylsilyl ethers. I. Aldopentoses. J Chromatogr. 398, 9-20 (1987).

42. P. M. Medeiros, B. R. T. Simoneit, Analysis of sugars in environmental samples by gas chromatography-mass spectrometry. J Chromatogr. A 1141, 271-278 (2007).

43. G. Petersson, Mass spectrometry of alditols as trimethylsilyl derivatives. Tetrahedron 25, 4437-4443 (1969).

44. G. Petersson, Mass spectrometry of aldonic and deoxyaldonic acids as trimethylsilyl derivatives. Tetrahedron 26, 3413-3428 (1970). 
45. I. Molnár-Perl, A. Vasanits, K. Horváth, Simultaneous GC-MS quantitation of phosphoric, aliphatic and aromatic carboxylic acids, proline and hyrdroxymethylfurfurol as their trimethylsilyl derivatives: in model solutions II. Chromatographia 48, 111-119 (1998). 